\title{
Uma Versão Modificada do Método Evolução Diferencial para Estimativa de Parâmetros do Modelo de Difusão Anômala Assimétrica
}

\author{
Luciano G. da Silva, Diego C. Knupp, Antônio J. da Silva Neto, Leôncio D. T. \\ Câmara \\ Universidade do Estado do Rio de Janeiro, Instituto Politécnico \\ 28630-050 - Nova Friburgo, RJ \\ E-mail: luciano@iprj.uerj.br, diegoknupp@gmail.com, ajsneto@iprj.uerj.br, dcamara@iprj.uerj.br
}

\author{
Luiz Bevilacqua \\ Universidade Federal do Rio de Janeiro, COPPE/UFRJ \\ 21941-972 - Rio de Janeiro, RJ \\ E-mail: bevilacqua@coc.ufrj.br \\ Augusto C. N. R. Galeão \\ Laboratório Nacional de Computação Científica \\ 25651-076 - Petrópolis, RJ \\ E-mail: acng@lncc.br \\ Orestes, L. Santiago \\ Instituto Superior Jose Antonio Echeverría, CUJAE \\ 19390 - Marianao, Havana, Cuba \\ E-mail: orestes@electrica.cujae.cu
}

\begin{abstract}
Resumo: No contexto de solução de problemas inversos, formulados implicitamente, os métodos de otimização estocásticos desempenham um papel fundamental para a estimativa de parâmetros. Entre esses métodos, destaca-se o algoritmo Evolução Diferencial (Differential Evolution - DE). Neste trabalho, é apresentada uma versão modificada deste método para estimar parâmetros de um novo modelo para o fenômeno de difusão anômala assimétrica. Os resultados apresentados neste trabalho indicam que com esta modificação, é possível diminuir o tempo de CPU através da redução do número de iterações empregando uma redução do espaço de busca para cada incógnita.
\end{abstract}

\section{INTRODUÇÃO}

O uso de técnicas de otimização estocásticas, em larga escala, foi possibilitado, principalmente, pelo avanço tecnológico computacional. No entanto, geralmente estas estratégias apresentam um elevado tempo de CPU, principalmente na solução de problemas inversos que, dependendo da formulação, requerem uma solução numérica para o problema direto. O algoritmo Evolução Diferencial (Differential Evolution - DE) [10, 13], é um método muito conhecido devido seu desempenho em funções de testes de otimização. Neste trabalho, é apresentada uma modificação do DE com aplicação na estimativa de parâmetros a partir da solução do problema inverso para um novo modelo de difusão anômala assimétrica [5], onde um parâmetro de controle $\beta$, que representa a taxa de redistribuição das partículas, é introduzido para impedir a ocorrência isolada do fenômeno de difusão. $\mathrm{O}$ objetivo da aplicação desta modificação no DE é a redução significativa do tempo de CPU neste problema, de forma a manter a qualidade de estimação.

O modelo de difusão anômala com distribuição assimétrica, corresponde ao clássico modelo de advecção-difusão. A diferença entre a clássica formulação e o novo modelo derivado da distribuição assimétrica está na preservação do parâmetro de controle para a taxa de redistribuição das partículas, o que mantém o significado original da intensidade relativa da difusão e propagação [5]. Em fenômenos de transporte, há uma série de aplicações onde a equação de advecção-difusão serve de modelo, podemos citar por exemplo, modelos cinéticos multicomponentes em processos biotecnológicos [3,6-9]. Na seção seguinte, apresentam-se as principais equações para o modelo de difusão anômala. 


\section{PROBLEMA DIRETO - MODELO DE DIFUSÃO ANÔMALA}

\section{Distribuição Simétrica}

Um novo modelo para a difusão anômala [4] foi proposto a partir de fenômenos em dinâmicas de população. Neste modelo um parâmetro de controle $\beta$ representa a fração de partículas capazes de se difundir, distribuída da seguinte forma: uma fração de partículas $\beta / 2$ é transferida para a célula da esquerda, $\beta / 2$ para a célula da direita, enquanto que a fração $(1-\beta)$ é retida na célula $n$. O desenvolvimento deste modelo pode ser visto em $[4,5]$. Este mecanismo, resulta no seguinte modelo simétrico para a difusão anômala

$$
\frac{\partial p}{\partial t}=\beta K_{1} \frac{\partial^{2} p}{\partial x^{2}}-\beta K_{2}(1-\beta) \frac{\partial^{4} p}{\partial x^{4}}
$$

onde $p$ representa a concentração de partículas, $K_{1}$ e $K_{2}$ são parâmetros derivados de fatores de escala. Em 2013 e 2014, Silva e colaboradores [11, 12] empregaram técnicas estocásticas na solução do problema inverso para estimativa dos parâmetros apresentados neste modelo.

\section{Distribuição Assimétrica (Problema Direto)}

Para a migração não-simétrica entre as células, assume-se que não há retenção [5], desta forma, obtém-se uma reprodução da clássica formulação de difusão-advecção.

$$
\frac{\partial p}{\partial t}=K_{2}\left(1-\beta^{2}\right) \frac{\partial^{2} p}{\partial x^{2}}-K_{1} \beta \frac{\partial p}{\partial x}
$$

onde $-1 \leq \beta \leq 1$. Para $\beta=0$ o problema é reduzido à formulação clássica de difusão.

Um importante resultado neste modelo, é a preservação explícita do parâmetro de controle $\beta$ na descrição matemática da Eq. (2). Para $\beta= \pm 1$ a propagação prevalece para a direita ou para à esquerda, e para $\beta=0$ a difusão simétrica domina [5]. Para o caso aqui apresentado a velocidade de fluxo sobreposta ao processo de difusão é igual a $\beta$, fator de desequilíbrio no processo de redistribuição. Os detalhes do desenvolvimento da Eq. (2) pode ser consultado em [5]. Para a solução desta equação, empregou-se o método numérico de diferenças finitas.

\section{FORMULAÇÃO DO PROBLEMA INVERSO}

Considerando que, na Eq. (2), não se tenha conhecimento do conjunto de parâmetros $Z=\left\{\beta, K_{1}, K_{2}\right\}$ e que se tenha, porém, valores disponíveis da variável observável $p$, medidos experimentalmente. Seja $Q(\boldsymbol{Z})$ o somatório dos resíduos quadrados, dados por:

$$
Q(\boldsymbol{Z})=\sum_{l=1}^{N_{d}}\left[p_{l}^{\text {calc }}(\boldsymbol{Z})-p_{l}^{\text {exp }}\right]^{2}
$$

onde $p^{\text {calc }}(\boldsymbol{Z})$ é o valor da variável observável calculada em $\boldsymbol{Z}, p_{l}^{\text {calc }}$ é o valor da concentração medida experimentalmente, $N_{d}$ o número de dados experimentais disponíveis. A solução da Eq. (3) pode ser interpretada como um problema de otimização, onde busca-se o valor de $\boldsymbol{Z}$ que minimize o funcional $Q(Z)$. Esta é a formulação implícita para o problema inverso.

\section{Algoritmo Evolução Diferencial (Differential Evolution - DE)}

O Evolução Diferencial (Differential Evolution - DE) [10,13] é um método estocástico de otimização, inspirado na evolução das espécies, que consiste na realização de operações vetoriais na qual a diferença ponderada entre dois indivíduos distintos, adicionada a um terceiro indivíduo, leva à geração de novos candidatos para a solução do problema de otimização. De forma geral, o DE apresenta as operações: inicialização da população, mutação, cruzamento e seleção, além de um critério de parada definido a priori. 


\section{Evolução Diferencial Modificado}

Esta modificação do DE, foi inspirada no método da seção áurea [1] e consiste na redução do espaço de busca de cada incógnita a cada vez que se tenha uma redução no valor da função objetivo $Q(\boldsymbol{Z})$ durante o processo iterativo. Considerando um total de $N_{i}$ incógnitas, e sejam $U(j)$ e $L(j), j=1,2, \ldots, N_{i}$, respectivamente, os limites superior e inferior de busca para cada incógnita $Z_{k}^{j}$, definimos um novo limite $a(j)$ dado por

$$
a(j)=(U(j)+L(j)) \cdot \phi
$$

onde $\phi=0.618$ corresponde a um valor aproximado para a razão áurea. A cada iteração $k$ é realizado o seguinte teste:

- Se $Q\left(Z_{\boldsymbol{k}}^{\text {melhor }}\right)<Q\left(Z_{\boldsymbol{k}-\boldsymbol{1}}^{\text {melhor }}\right)$

Se $Z_{k, j}^{\text {melhor }}<a(j)$ então $U(j)=a(j) \quad j=1,2, \ldots, N_{i}$

Se $Z_{k, j}^{\text {melhor }}>a(j)$ então $L(j)=a(j) \quad j=1,2, \ldots, N_{i}$

este procedimento iterativo é ilustrado na Figura 2, enquanto que na Figura 1, são apresentados os algoritmos para os métodos DE e DEmod.

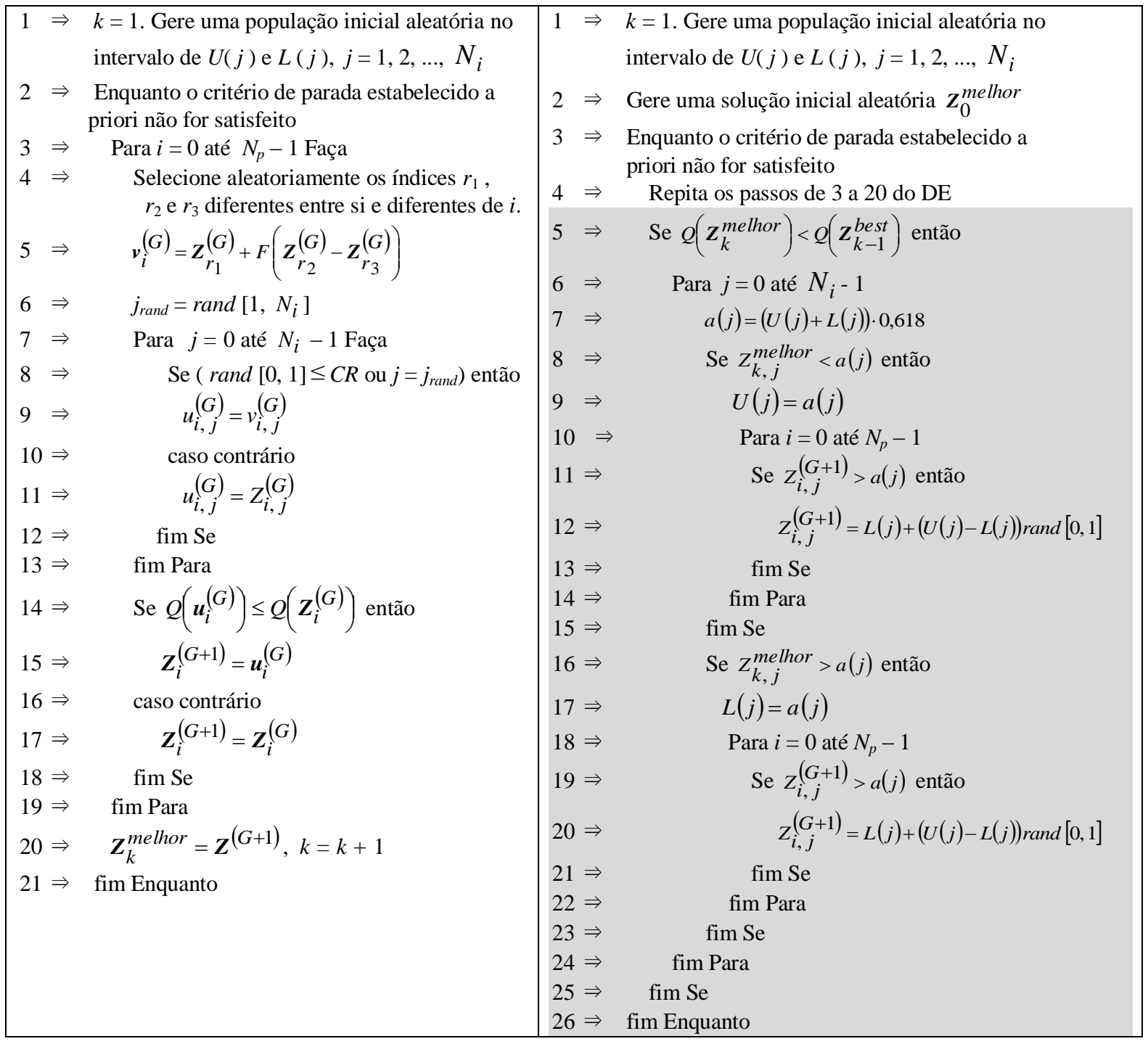

(a)

(b)

Figura 1 - Algoritmos numéricos (a) DE ; (b) DEmod 


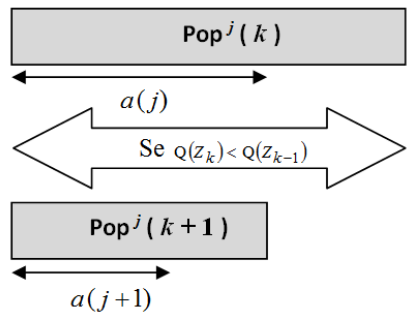

Figura 2 - Representação esquemática da redução dos limites de busca.

onde $N_{p}$ corresponde ao número de elementos da população, $N_{i}$ o número de incógnitas, $C R$ a probabilidade de cruzamento definida a priori, $U(j)$ e $L(j)$ são respectivamente os limites inferiores e superiores da $j$-ésima variável.

\section{Análise de Sensibilidade}

Uma questão que deve ser levada em consideração quando se usa dados experimentais reais ou sintéticos, refere-se à qualidade desses dados. A análise de sensibilidade pode contribuir para a escolha de dados experimentais com maior relevância na solução do problema inverso. Além disso, serve também para verificar possíveis correlações entre parâmetros, i.e. quando duas ou mais curvas dos coeficientes de sensibilidade apresentam mesma forma, ou forma semelhante, estes parâmetros podem estar correlacionados, afetando a unicidade da solução do problema inverso através de uma dependência linear entre estes parâmetros. Os coeficientes de sensibilidade são definidos por [2]:

$$
\bar{X}_{Z l}^{j}=\frac{\partial p_{\text {calc }}(\boldsymbol{Z})}{\partial Z_{l}}, j=1,2, \ldots, N_{d} \text { e } l=1,2, \ldots, N_{i}
$$

onde $N_{d}$ é o número de dados experimentais disponíveis e $N_{i}$ o número de incógnitas.

É comum o uso dos coeficientes de sensibilidade modificados, onde a Eq. (5) é multiplicada pela própria incógnita e, desta forma, os coeficientes passam a ter a unidade de medida da variável observável, ou seja

$$
X_{Z_{l}}^{j}=Z_{l} \cdot \bar{X}_{Z_{l}}^{j}=Z_{l} \cdot \frac{\partial p_{\text {calc }}(\boldsymbol{Z})}{\partial Z_{l}}, \quad j=1,2, \ldots, N_{d} \text { e } l=1,2, \ldots, N_{i}
$$

\section{RESULTADOS}

\section{Problema Direto}

Para a solução do problema direto usou-se o seguinte conjunto de parâmetros

\begin{tabular}{c|c|c}
\hline$\beta$ & $K_{1}$ & $K_{2}$ \\
\hline 0,2 & 0,01 & 0,001 \\
\hline
\end{tabular}

Tabela 1 - Parâmetros empregados na solução do problema direto, Eq.(2).

Na Figura 3 são apresentadas as curvas para a solução do problema direto, para os tempos $t=0 ; 200,500,1000,2000$ e $0 \leq x \leq 10$, empregando as seguintes condições de contorno e inicial

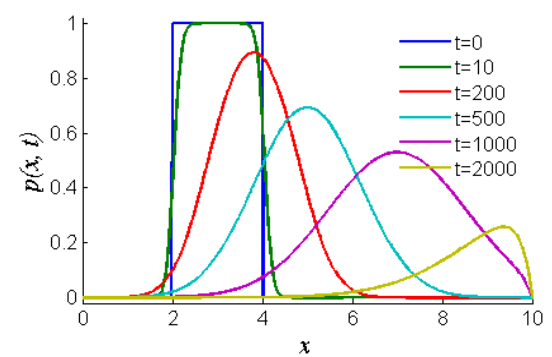

Figura 3 - Solução do problema direto empregando o método de diferenças finitas.

$$
p(0, t)=p(10, t)=0 \quad \text { para } t>0
$$




$$
f(x)=\left\{\begin{array}{ll}
1, & \text { se } \quad 2 \leq x \leq 4 \\
0, & \text { se } \quad x<2 \text { ou } x>4
\end{array} \text { para } t=0, \quad 0 \leq x \leq 10\right.
$$

onde $N$ é o número de nós empregados na malha computacional de diferenças finitas.

\section{Resultados para a Análise de Sensibilidade}

Os coeficientes de sensibilidade apresentados nesta seção foram obtidos na posição $x=3$ até o instante de tempo $t=2000$, usando a Eq.(6). Na Figura 4 são apresentados os coeficientes de sensibilidade $X_{\beta}, X_{K_{1}}$ e $X_{K_{2}}$ para os parâmetros da Eq. (2).

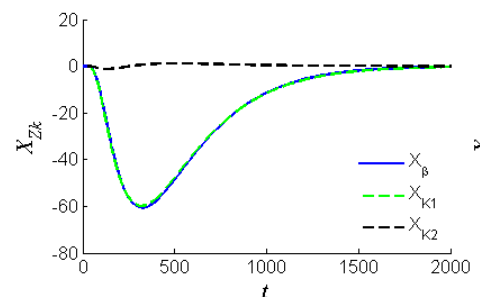

(a)

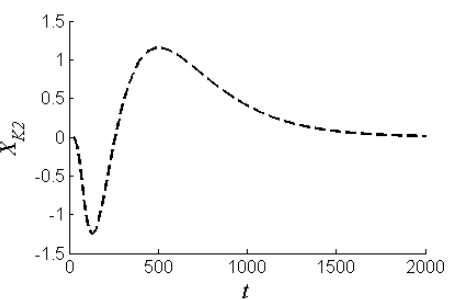

(b)

Figura 4 - (a) Coeficientes de sensibilidade $X_{Z_{k}}$ (b) Coeficiente de sensibilidade $X_{K_{2}}$.

De acordo com a Figura 4a, as curvas de sensibilidade $X_{\beta}$ e $X_{K_{1}}$ são essencialmente coincidentes, o que fisicamente significa que perturbações nos valores destes parâmetros resultam em variações idênticas na variável observável do problema, $p$, ou seja, infinitas combinações destes dois parâmetros levam à mesma resposta do problema. Na prática, a obtenção de uma solução única do problema inverso para estimativa simultânea destes dois parâmetros é impossível [2], sendo necessário, portanto, informações prévias sobre o parâmetro $\beta$ ou o parâmetro $K_{1}$. Além disso, verifica-se que a curva de sensibilidade $X_{K_{2}}$ apresenta valores muito próximos de zero em comparação com as duas outras curvas de sensibilidade, sendo apresentada esta mesma curva em outra escala gráfica, na Figura $4 b$, de modo a ilustrar melhor sua variação no tempo. Os baixos valores do coeficiente $X_{K_{2}}$ significam uma baixa sensibilidade da variável observável $p$ com relação ao parâmetro $K_{2}$, o que na prática resulta em uma maior dificuldade na solução do problema inverso para estimativa deste parâmetro [2]. Com base nisto, foram introduzidos dois novos parâmetros $\alpha_{1}=K_{2}\left(1-\beta^{2}\right)$ e $\alpha_{2}=\beta K_{1}$, desta forma, a Eq.(2) passa ser escrita como

$$
\frac{\partial p}{\partial t}=\alpha_{1} \frac{\partial^{2} p}{\partial x^{2}}-\alpha_{2} \frac{\partial p}{\partial x}
$$

e, consequentemente, tem-se os respectivos coeficientes de sensibilidade para $\alpha_{1}$ e $\alpha_{2}$

$$
X_{\alpha_{1}}=\alpha_{1} \frac{\partial p}{\partial \alpha_{1}}, \quad X_{\alpha_{2}}=\alpha_{2} \frac{\partial p}{\partial \alpha_{2}}
$$

cujas curvas são apresentadas na Figura 5.

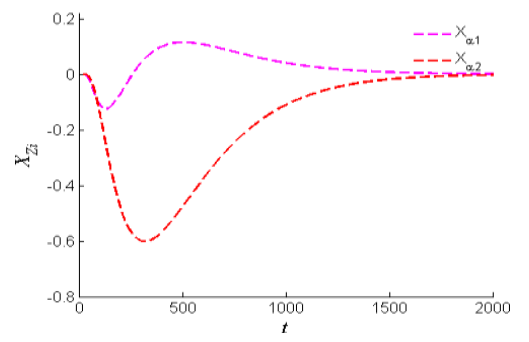

Figura 5 - Curvas para os coeficientes de sensibilidade $X_{\alpha_{1}}$ e $X_{\alpha_{2}}$.

Observa-se na Figura 5, que de acordo com os coeficientes de sensibilidade $X_{\alpha_{1}}$ e $X_{\alpha_{2}}$, os parâmetros $\alpha_{1}$ e $\alpha_{2}$ não estão linearmente correlacionados, o que significa que é possível 
estimar simultaneamente estes dois parâmetros. Na seção seguinte são apresentados os resultados para a estimativa simultânea de $\alpha_{1}$ e $\alpha_{2}$ empregando os métodos estocásticos DE e DEmod.

\section{Resultados para o Problema Inverso}

Finalmente, apresenta-se nas Tabelas 2 e 3 os resultados obtidos para a solução do problema inverso tratado neste trabalho, ou seja, para a estimativa simultânea de $\alpha_{1}$ e $\alpha_{2}$, usando os valores dados na Tabela 1 para $\beta, K_{1}$ e $K_{2}$, i.e. $\alpha_{1}=9,6 \times 10^{-4}$ e $\alpha_{2}=2 \times 10^{-3}$. Para a simulação do problema inverso, foram usados dados experimentais sintéticos considerando um ruído gerado a partir de uma distribuição Normal com média zero e desvio padrão constante, dados por

$$
p_{i}^{\text {exp }}=p_{i}^{\text {calc }}\left(\boldsymbol{Z}_{\text {exato }}\right)+\varepsilon_{i} \text {, onde } \varepsilon \sim N\left(0, \sigma_{e}\right)
$$

Nos resultados apresentados a seguir, empregou-se $\sigma_{e}=0,02$ na Eq. (12), o que corresponde a erros de aproximadamente $4 \%$ nos dados experimentais, $p_{i}^{\exp }$, obtidos na posição $x=3$ e nos tempos $t=300,301, \ldots, 399$. Utilizou-se o seguinte critério de parada: $K_{\max }=50$ iterações ou até que o valor da função objetivo seja menor que $7 \times 10^{-3}$, onde $K_{\max }$ é o número máximo de iterações. Todos os resultados apresentados neste trabalho foram obtidos através de um computador DELL LATITUDE E6500, 8GB de RAM e HD de 300GB, processador Intel ${ }^{\circledR}$ Core $^{\mathrm{TM}} 2$ Duo CPU T9600@2.80GHz com sistema operacional Windows 764 Bits.

\begin{tabular}{ccccc}
\hline $\mathrm{Q}(\mathbf{Z})$ & $\alpha_{1}=9,6 \times 10^{-4}$ & $\alpha_{2}=2 \times 10^{-3}$ & Iterações & Tempo (s) \\
\hline $6,293959 \times 10^{-3}$ & $9,547549 \times 10^{-4}$ & $2,003531 \times 10^{-3}$ & 46 & 92 \\
$6,650341 \times 10^{-3}$ & $1,200448 \times 10^{-3}$ & $2,053876 \times 10^{-3}$ & 45 & 95 \\
$6,289184 \times 10^{-3}$ & $1,086108 \times 10^{-3}$ & $2,023902 \times 10^{-3}$ & 45 & 91 \\
$6,518802 \times 10^{-3}$ & $1,056584 \times 10^{-3}$ & $2,034937 \times 10^{-3}$ & 49 & 97 \\
$5,949596 \times 10^{-3}$ & $8,788786 \times 10^{-4}$ & $1,984420 \times 10^{-3}$ & 40 & 87 \\
$6,251918 \times 10^{-3}$ & $1,131917 \times 10^{-3}$ & $2,035654 \times 10^{-3}$ & 45 & 87 \\
$6,634266 \times 10^{-3}$ & $1,045641 \times 10^{-3}$ & $2,027464 \times 10^{-3}$ & 46 & 93 \\
$6,885171 \times 10^{-3}$ & $1,044311 \times 10^{-3}$ & $2,028658 \times 10^{-3}$ & 46 & 103 \\
$6,719908 \times 10^{-3}$ & $1,031870 \times 10^{-3}$ & $2,028189 \times 10^{-3}$ & 41 & 78 \\
$7,557647 \times 10^{-3}$ & $1,290794 \times 10^{-3}$ & $2,085256 \times 10^{-3}$ & 50 & 104 \\
\hline Média & $1,072131 \times 10^{-3}$ & $2,030589 \times 10^{-3}$ & $\mathbf{4 5 , 3 0}$ & $\mathbf{9 2 , 7 0}$ \\
DP & $1,166716 \times 10^{-4}$ & $2,688041 \times 10^{-5}$ & 3,06 & 7,76 \\
\hline
\end{tabular}

Tabela 2 - Evolução Diferencial Clássico com até 4\% de ruído. $\mathrm{Nd}=40$.

\begin{tabular}{ccccc}
\hline $\mathrm{Q}(\mathbf{Z})$ & $\alpha_{1}=9,6 \times 10^{-4}$ & $\alpha_{2}=2 \times 10^{-3}$ & Iterações & Tempo (s) \\
\hline $6,376027 \times 10^{-3}$ & $8,366427 \times 10^{-4}$ & $1,963260 \times 10^{-3}$ & 27 & 49 \\
$4,984042 \times 10^{-3}$ & $9,441667 \times 10^{-4}$ & $1,998424 \times 10^{-3}$ & 27 & 48 \\
$5,868172 \times 10^{-3}$ & $9,983130 \times 10^{-4}$ & $2,015076 \times 10^{-3}$ & 32 & 57 \\
$6,704496 \times 10^{-3}$ & $1,191015 \times 10^{-3}$ & $2,058249 \times 10^{-3}$ & 24 & 52 \\
$6,069629 \times 10^{-3}$ & $1,102267 \times 10^{-3}$ & $2,027851 \times 10^{-3}$ & 25 & 44 \\
$6,372619 \times 10^{-3}$ & $1,136756 \times 10^{-3}$ & $2,038961 \times 10^{-3}$ & 26 & 45 \\
$4,944204 \times 10^{-3}$ & $9,887445 \times 10^{-4}$ & $2,000655 \times 10^{-3}$ & 27 & 47 \\
$6,831348 \times 10^{-3}$ & $1,060137 \times 10^{-3}$ & $2,030219 \times 10^{-3}$ & 32 & 56 \\
$6,617386 \times 10^{-3}$ & $1,253180 \times 10^{-3}$ & $2,063247 \times 10^{-3}$ & 31 & 54 \\
$6,814377 \times 10^{-3}$ & $9,623642 \times 10^{-4}$ & $2,007292 \times 10^{-3}$ & 43 & 87 \\
\hline Média & $1,047359 \times 10^{-3}$ & $2,020323 \times 10^{-3}$ & $\mathbf{2 9 , 4 0}$ & $\mathbf{5 3 , 9 0}$ \\
DP & $1,257355 \times 10^{-4}$ & $3,000509 \times 10^{-5}$ & 5,56 & 12,45 \\
\hline
\end{tabular}

Tabela 3 - Evolução Diferencial Modificado com até $4 \%$ de ruído. $\mathrm{Nd}=40$.

\section{CONCLUSÕES}

Verifica-se nas tabelas 2 e 3, que o método DEmod apresentou um desempenho melhor que o $\mathrm{DE}$, tanto no número de iterações quanto no custo computacional. Isso mostra o sucesso obtido pela estratégia aqui adotada, no caso, a redução do espaço de busca usando a razão áurea. 
Foi possível estimar valores aproximados para os parâmetros $\alpha_{1}$ e $\alpha_{2}$, confirmando o que havia sido observado através da análise de sensibilidade, Figura 5, i.e. estes parâmetros não estão correlacionados, possibilitando a estimativa simultânea empregando a solução do problema inverso. A média e o desvio padrão, tanto no DE quanto no DEmod, revelam a unicidade para a solução deste problema considerando os parâmetros $\alpha_{1}$ e $\alpha_{2}$. Durante o processo de redução do espaço de busca, uma limitação que pode ser apresentada pelo DEmod é a eliminação do espaço de busca que contém o mínimo global, portanto, para o emprego desta modificação do DE em trabalhos futuros, sugere-se uma alteração do critério de redução, por exemplo, reduzir o espaço de busca a partir de um certo número de iterações.

\section{Agradecimentos}

Os autores agradecem à Coordenação de Aperfeiçoamento de Pessoal de Nível Superior (CAPES) pelo apoio financeiro que permitiram a realização deste trabalho.

\section{Referências}

[1] Bazaraa, M., Sherali, H. D.; Sketty, C. M. Nonlinear Programming: Theory and Algorithms, 3rd ed. New Jersey: Wiley Interscience, 2006.

[2] Beck, J. V., Blackwell, B. and St. Clair Jr., C. R., Inverse heat conduction - Ill-Posed Problems, Wiley-Interscience, 1985.

[3] Bellot, J. C. , Condoret, J. S., Liquid chromatography codelling: A Review. Process Biochemistry, 26:363, 1991

[4] Bevilacqua, L., Galeão, A. C. N. R. and Costa, F. P., A new analytical formulation of retention effects on particle diffusion process, Annals of the Brazilian Academy of Sciences, Vol. 83, No. 4, pp. 1443-1464, 2011a.

[5] Bevilacqua, L., Galeão, A. C. N. R. and Costa, F. P., On the significance of higher order differential terms in diffusion processes. Journal of the Brazilian Society of Mechanical Sciences and Engineering, v. 34, p. 166-175, $2011 \mathrm{~b}$.

[6] Gu, T., Tsai G. J., Tsao, G.T., New approach to a general nonlinear multicomponent chromatography model. AIChE J 36:784, 1990.

[7] Gu, T., Tsai, G. J. , Tsao, G. T., Ladish, M. R., Displacement effect in multicomponent chromatography. AIChE J 36:1156, 1990.

[8] Gu, T., Tsai, G. J. and Tsao, G. T., "Study of multicomponent adsorption and chromatography with uneven saturation capacities." AIChE J., 37(9), pp.1333-1340, 1991.

[9] Gu, T., Mathematical Modeling and Scale-up of Liquid Chromatography. Springer, Berlin, 1995.

[10] Price, K., Storn, R., Lampinem, J. A. Differential Evolution : A Practical Approach to Global Optimization. Berlin Heidelberg: Springer-Verlag, 2006.

[11] Silva, L. G., Knupp, D. C., Bevilacqua, L., Galeão, A. C. N. R., Simas, J. G., Vasconcellos, J. F., Silva Neto, A. J., Investigation of a new model for anomalous diffusion phenomena by means of an inverse analysis, $4^{\text {th }}$ Inverse Problems, Design and Optimization Symposium (IPDO-2013) Albi, France, June 26-28, 2013.

[12] Silva, L. G., Knupp, D. C., Bevilacqua, L., Galeão, A. C. N. R., , Silva Neto, A. J., Formulação e solução de um problema inverso de difusão anômala com técnicas estocásticas, Ciência e Natura, Aceito para publicação, (2014).

[13] Storn, R., Price, K. Differential Evolution: a simple and efficient adaptive scheme for global optimization over continuous spaces, International Computer Science Institute, Technical Report TR-95-012, pp. 1 - 15, 1995. 